PROCEEDINGS OF THE

AMERICAN MATHEMATICAL SOCIETY

Volume 138, Number 1, January 2010, Pages 37-46

S 0002-9939(09)10067-9

Article electronically published on September 4, 2009

\title{
CONGRUENCES \\ FOR THE SECOND-ORDER CATALAN NUMBERS
}

\author{
LI-LU ZHAO, HAO PAN, AND ZHI-WEI SUN
}

(Communicated by Ken Ono)

Abstract. Let $p$ be any odd prime. We mainly show that

$$
\sum_{k=1}^{p-1} \frac{2^{k}}{k}\left(\begin{array}{c}
3 k \\
k
\end{array}\right) \equiv 0(\bmod p)
$$

and

$$
\sum_{k=1}^{p-1} 2^{k-1} C_{k}^{(2)} \equiv(-1)^{(p-1) / 2}-1(\bmod p),
$$

where $C_{k}^{(2)}=\left(\begin{array}{c}3 k \\ k\end{array}\right) /(2 k+1)$ is the $k$ th Catalan number of order 2 .

\section{InTRODUCTION}

The well-known Catalan numbers are those integers

$$
C_{n}=\frac{1}{n+1}\left(\begin{array}{c}
2 n \\
n
\end{array}\right)=\left(\begin{array}{c}
2 n \\
n
\end{array}\right)-\left(\begin{array}{c}
2 n \\
n-1
\end{array}\right) \quad(n=0,1,2, \ldots)
$$

(As usual we regard $\left(\begin{array}{c}x \\ -k\end{array}\right)$ as 0 for $k=1,2, \ldots$ ) There are many combinatorial interpretations for these important numbers (see, e.g., [St, pp. 219-229]). With the help of a sophisticated binomial identity, H. Pan and Z. W. Sun [PS] obtained some congruences on sums of Catalan numbers; in particular, by $\underline{\mathrm{PS}}$, (1.16) and (1.8)], for any prime $p>3$ we have

$$
\sum_{k=0}^{p-1} C_{k} \equiv \frac{3\left(\frac{p}{3}\right)-1}{2}(\bmod p) \text { and } \sum_{k=1}^{p-1} \frac{C_{k}}{k} \equiv \frac{3}{2}\left(1-\left(\frac{p}{3}\right)\right)(\bmod p),
$$

where the Legendre symbol $\left(\frac{a}{3}\right) \in\{0, \pm 1\}$ satisfies the congruence $a \equiv\left(\frac{a}{3}\right)(\bmod 3)$. Recently Z. W. Sun and R. Tauraso ST1, ST2] obtained some further congruences concerning sums involving Catalan numbers.

For $m, n \in \mathbb{N}=\{0,1,2, \ldots\}$, we define

$$
C_{n}^{(m)}=\frac{1}{m n+1}\left(\begin{array}{c}
m n+n \\
n
\end{array}\right)=\left(\begin{array}{c}
m n+n \\
n
\end{array}\right)-m\left(\begin{array}{c}
m n+n \\
n-1
\end{array}\right)
$$

Received by the editors April 7, 2009.

2000 Mathematics Subject Classification. Primary 11B65; Secondary 05A10, 11A07.

The third author is the corresponding author. He was supported by the National Natural Science Foundation (grant 10871087) and the Overseas Cooperation Fund of China.

(C)2009 American Mathematical Society Reverts to public domain 28 years from publication 
and call it the $n$th Catalan number of order $m$. Clearly

$$
C_{n}^{(1)}=C_{n} \quad \text { and } \quad C_{n}^{(2)}=\frac{1}{2 n+1}\left(\begin{array}{c}
3 n \\
n
\end{array}\right) .
$$

In contrast with (1.0), we have the following result involving the second-order Catalan numbers.

Theorem 1.1. Let $p$ be an odd prime. Then

$$
\sum_{k=1}^{p-1} 2^{k} C_{k}^{(2)} \equiv 2\left((-1)^{(p-1) / 2}-1\right)(\bmod p)
$$

and

$$
\sum_{k=1}^{p-1} \frac{2^{k} C_{k}^{(2)}}{k} \equiv 4\left(1-(-1)^{(p-1) / 2}\right)(\bmod p) .
$$

Actually Theorem 1.1 follows from our next two theorems.

Theorem 1.2. Let $p>5$ be a prime. Then

$$
\begin{aligned}
\sum_{k=0}^{p-1} 2^{k}\left(\begin{array}{c}
3 k \\
k
\end{array}\right) & \equiv \frac{6(-1)^{(p-1) / 2}-1}{5}(\bmod p), \\
\sum_{k=0}^{p-1} 2^{k}\left(\begin{array}{c}
3 k+1 \\
k
\end{array}\right) & \equiv \frac{4(-1)^{(p-1) / 2}+1}{5}(\bmod p) .
\end{aligned}
$$

Theorem 1.3. For any prime $p$ we have

$$
\sum_{k=1}^{p-1} \frac{2^{k}}{k}\left(\begin{array}{c}
3 k \\
k
\end{array}\right) \equiv 0(\bmod p) .
$$

For any odd prime $p$ we can also prove the following congruences:

$$
\begin{aligned}
& 5 \sum_{k=1}^{p-1} 2^{k}\left(\begin{array}{c}
3 k+2 \\
k
\end{array}\right) \equiv(-1)^{(p-1) / 2}-1(\bmod p), \\
& \sum_{k=1}^{p-1} \frac{2^{k-1}}{k}\left(\begin{array}{c}
3 k+1 \\
k
\end{array}\right) \equiv(-1)^{(p-1) / 2}-1(\bmod p), \\
& \sum_{k=1}^{p-1} \frac{2^{k-1}}{k}\left(\begin{array}{c}
3 k+2 \\
k
\end{array}\right) \equiv \frac{3}{2}\left((-1)^{(p-1) / 2}-1\right)(\bmod p) .
\end{aligned}
$$

We omit their proofs, which are similar to those of Theorems 1.2 and 1.3.

With the help of Theorems 1.2 and 1.3, we can easily deduce Theorem 1.1.

Proof of Theorem 1.1 via Theorems 1.2 and 1.3. Clearly (1.1) and (1.2) hold for $p=3,5$. Assume $p>5$. By (1.3) and (1.4),

$$
\begin{aligned}
\sum_{k=0}^{p-1} \frac{2^{k}}{2 k+1}\left(\begin{array}{c}
3 k \\
k
\end{array}\right) & =3 \sum_{k=0}^{p-1} 2^{k}\left(\begin{array}{c}
3 k \\
k
\end{array}\right)-2 \sum_{k=0}^{p-1} 2^{k}\left(\begin{array}{c}
3 k+1 \\
k
\end{array}\right) \\
& \equiv 2(-1)^{(p-1) / 2}-1(\bmod p) .
\end{aligned}
$$


This proves (1.1). For (1.2) it suffices to note that

$$
\sum_{k=1}^{p-1} \frac{2^{k}}{k(2 k+1)}\left(\begin{array}{c}
3 k \\
k
\end{array}\right)=\sum_{k=1}^{p-1} \frac{2^{k}}{k}\left(\begin{array}{c}
3 k \\
k
\end{array}\right)-2 \sum_{k=1}^{p-1} \frac{2^{k}}{2 k+1}\left(\begin{array}{c}
3 k \\
k
\end{array}\right) .
$$

This concludes the proof.

We are going to provide two lemmas in the next section. Theorems 1.2 and 1.3 will be proved in Sections 3 and 4 respectively.

\section{Some LEMmas}

Lemma 2.1. For $m, n \in \mathbb{N}$ we have

$$
\begin{aligned}
& 2^{n} \sum_{k=0}^{\lfloor m / 3\rfloor}(-2)^{k}\left(\begin{array}{c}
n \\
m-3 k
\end{array}\right)\left(\begin{array}{c}
3 k-m+n \\
k
\end{array}\right) \\
= & (-1)^{m} \sum_{j=0}^{n}\left(\begin{array}{c}
n \\
j
\end{array}\right) \sum_{k=0}^{m}(-2)^{k}\left(\begin{array}{c}
n \\
m-k
\end{array}\right)\left(\begin{array}{c}
2 j \\
k
\end{array}\right) .
\end{aligned}
$$

Proof. Let $P(x)=\left(2+2 x-4 x^{3}\right)^{n}$, and denote by $\left[x^{k}\right] P(x)$ the coefficient of $x^{k}$ in the expansion of $P(x)$. Then

$$
\begin{aligned}
2^{-n}\left[x^{m}\right] P(x) & =\left[x^{m}\right]\left((1+x)-2 x^{3}\right)^{n} \\
& =\sum_{k=0}^{\lfloor m / 3\rfloor}\left(\begin{array}{l}
n \\
k
\end{array}\right)(-2)^{k}\left[x^{m-3 k}\right](1+x)^{n-k} \\
& =\sum_{k=0}^{\lfloor m / 3\rfloor}(-2)^{k}\left(\begin{array}{l}
n \\
k
\end{array}\right)\left(\begin{array}{c}
n-k \\
m-3 k
\end{array}\right) \\
& =\sum_{k=0}^{\lfloor m / 3\rfloor}(-2)^{k}\left(\begin{array}{c}
n \\
m-3 k
\end{array}\right)\left(\begin{array}{c}
3 k-m+n \\
k
\end{array}\right) .
\end{aligned}
$$

Since

$$
P(x)=(1-x)^{n}\left((2 x+1)^{2}+1\right)^{n}=\sum_{j=0}^{n}\left(\begin{array}{l}
n \\
j
\end{array}\right)(1-x)^{n}(2 x+1)^{2 j},
$$

we also have

$$
\left[x^{m}\right] P(x)=\sum_{j=0}^{n}\left(\begin{array}{c}
n \\
j
\end{array}\right) \sum_{k=0}^{m} 2^{k}\left(\begin{array}{c}
2 j \\
k
\end{array}\right)(-1)^{m-k}\left(\begin{array}{c}
n \\
m-k
\end{array}\right) .
$$

Therefore (2.1) is valid.

For any prime $p$, if $n, k \in \mathbb{N}$ and $s, t \in\{0,1, \ldots, p-1\}$, then we have the wellknown Lucas congruence (cf. $\mathrm{Gr}$ or $[\mathrm{HS}]),\left(\begin{array}{c}p n+s \\ p k+t\end{array}\right) \equiv\left(\begin{array}{l}n \\ k\end{array}\right)\left(\begin{array}{l}s \\ t\end{array}\right)(\bmod p)$. This will be used in the proof of the following lemma.

Lemma 2.2. Let $p>5$ be a prime. Then we have

$$
\sum_{s=0}^{p-1}(-1)^{s} \sum_{t=0}^{p-1} 2^{t}\left(\begin{array}{c}
2 s \\
t
\end{array}\right) \equiv \frac{3(-1)^{(p-1) / 2}+2}{5}(\bmod p)
$$


and

$$
\sum_{s=0}^{p-1}(-1)^{s} \sum_{t=0}^{p-1} 2^{t}\left(\begin{array}{c}
2 s \\
p+t
\end{array}\right) \equiv \frac{3}{10}\left(1-(-1)^{(p-1) / 2}\right)(\bmod p)
$$

Proof. Observe that

$$
\begin{aligned}
& \sum_{s=0}^{p-1}(-1)^{s} \sum_{t=0}^{p-1} 2^{t}\left(\begin{array}{c}
2 s \\
t
\end{array}\right) \\
= & \sum_{s=0}^{(p-1) / 2}(-1)^{s} \sum_{t=0}^{2 s} 2^{t}\left(\begin{array}{c}
2 s \\
t
\end{array}\right)+\sum_{s=(p+1) / 2}^{p-1}(-1)^{s} \sum_{t=0}^{p-1} 2^{t}\left(\begin{array}{c}
2 s \\
t
\end{array}\right) \\
= & \sum_{s=0}^{(p-1) / 2}(-1)^{s} 3^{2 s}+\sum_{s=(p+1) / 2}^{p-1}(-1)^{s} \sum_{t=0}^{p-1} 2^{t}\left(\begin{array}{c}
2 s \\
t
\end{array}\right) \\
= & \sum_{s=0}^{(p-1) / 2}(-1)^{s} 3^{2 s}+\sum_{s=(p+1) / 2}^{p-1}(-1)^{s}\left(\sum_{t=0}^{2 s} 2^{t}\left(\begin{array}{c}
2 s \\
t
\end{array}\right)-\sum_{t=p}^{2 s} 2^{t}\left(\begin{array}{c}
2 s \\
t
\end{array}\right)\right) \\
= & \sum_{s=0}^{p-1}(-1)^{s} 3^{2 s}-\sum_{s=(p+1) / 2}^{p-1}(-1)^{s} \sum_{t=p}^{2 s} 2^{t}\left(\begin{array}{c}
2 s \\
t
\end{array}\right) \\
= & \sum_{s=0}^{p-1}(-9)^{s}-\sum_{s=(p+1) / 2}^{p-1}(-1)^{s} \sum_{r=0}^{2 s-p} 2^{p+r}\left(\begin{array}{c}
2 s \\
p+r
\end{array}\right) .
\end{aligned}
$$

For $s=(p+1) / 2, \ldots, p-1$, by Lucas' congruence we have

$$
\sum_{r=0}^{2 s-p} 2^{r}\left(\begin{array}{c}
p+(2 s-p) \\
p+r
\end{array}\right) \equiv \sum_{r=0}^{2 s-p} 2^{r}\left(\begin{array}{c}
2 s-p \\
r
\end{array}\right)=3^{2 s-p}(\bmod p) .
$$

Thus, with the help of Fermat's little theorem, we get

$$
\begin{aligned}
\sum_{s=0}^{p-1}(-1)^{s} \sum_{t=0}^{p-1} 2^{t}\left(\begin{array}{c}
2 s \\
t
\end{array}\right) & \equiv \frac{1-(-9)^{p}}{10}-\sum_{s=(p+1) / 2}^{p-1}(-1)^{s} \frac{2}{3} \cdot 9^{s} \\
& \equiv 1-\frac{2}{3}(-9)^{\frac{p+1}{2}} \frac{1-(-9)^{(p-1) / 2}}{10} \\
& \equiv \frac{3(-1)^{(p-1) / 2}+2}{5}(\bmod p) .
\end{aligned}
$$

This proves (2.2). 
In view of Lucas' congruence and Fermat's little theorem, we also have

$$
\begin{aligned}
& \sum_{s=0}^{p-1}(-1)^{s} \sum_{t=0}^{p-1} 2^{t}\left(\begin{array}{c}
2 s \\
p+t
\end{array}\right) \\
\equiv & \sum_{s=(p+1) / 2}^{p-1}(-1)^{s} \sum_{t=0}^{p-1} 2^{t}\left(\begin{array}{c}
2 s-p \\
t
\end{array}\right)=\sum_{s=(p+1) / 2}^{p-1}(-1)^{s} 3^{2 s-p} \\
= & 3^{-p}(-9)^{(p+1) / 2} \frac{1-(-9)^{(p-1) / 2}}{10}=(-1)^{(p+1) / 2} \frac{3}{10}\left(1+(-1)^{(p+1) / 2} 3^{p-1}\right) \\
\equiv & \frac{3}{10}\left(1-(-1)^{(p-1) / 2}\right)(\bmod p) .
\end{aligned}
$$

So $(2.3)$ is also valid. We are done.

\section{Proof of Theorem 1.2}

In order to prove Theorem 1.2, we first present an auxiliary result.

Theorem 3.1. Let $p>5$ be a prime, and let $d, \delta \in\{0,1\}$. Then

$$
\begin{aligned}
& \frac{(-1)^{d+\delta}}{2^{\delta}} \sum_{\delta p-d \leqslant 3 k \leqslant \delta p+p-1-d} 2^{k}\left(\begin{array}{c}
3 k+d \\
k
\end{array}\right) \\
\equiv & \frac{4-\delta}{10}+\frac{(3 \delta-2)(5 d-3)}{10}(-1)^{(p-1) / 2}(\bmod p) .
\end{aligned}
$$

Proof. Applying (2.1) with $n=p-1$ and $m=\delta p+p-1-d$, we get

$$
\begin{aligned}
& 2^{p-1} \sum_{k=0}^{\lfloor(\delta p+p-1-d) / 3\rfloor}(-2)^{k}\left(\begin{array}{c}
p-1 \\
\delta p+p-1-d-3 k
\end{array}\right)\left(\begin{array}{c}
3 k+d-\delta p \\
k
\end{array}\right) \\
= & (-1)^{\delta p+p-1-d} \sum_{j=0}^{p-1}\left(\begin{array}{c}
p-1 \\
j
\end{array}\right) \sum_{k=0}^{\delta p+p-1-d}(-2)^{k}\left(\begin{array}{c}
p-1 \\
\delta p+p-1-d-k
\end{array}\right)\left(\begin{array}{c}
2 j \\
k
\end{array}\right) .
\end{aligned}
$$

Observe that

$$
\begin{aligned}
& \sum_{k=0}^{\lfloor(\delta p+p-1-d) / 3\rfloor}(-2)^{k}\left(\begin{array}{c}
p-1 \\
\delta p+p-1-d-3 k
\end{array}\right)\left(\begin{array}{c}
3 k+d-\delta p \\
k
\end{array}\right) \\
= & \sum_{\delta p-d \leqslant 3 k \leqslant \delta p+p-1-d}(-2)^{k}\left(\begin{array}{c}
p-1 \\
p+\delta p-1-d-3 k
\end{array}\right)\left(\begin{array}{c}
3 k+d-\delta p \\
k
\end{array}\right) \\
\equiv & \sum_{\delta p-d \leqslant 3 k \leqslant \delta p+p-1-d}(-2)^{k}(-1)^{\delta p+p-1-d-3 k}\left(\begin{array}{c}
3 k+d \\
k
\end{array}\right) \\
\equiv & (-1)^{d+\delta} \sum_{\delta p-d \leqslant 3 k \leqslant \delta p+p-1-d} 2^{k}\left(\begin{array}{c}
3 k+d \\
k
\end{array}\right)(\bmod p)
\end{aligned}
$$


and

$$
\begin{aligned}
& (-1)^{\delta p+p-1-d} \sum_{j=0}^{p-1}\left(\begin{array}{c}
p-1 \\
j
\end{array}\right) \sum_{k=0}^{\delta p+p-1-d}(-2)^{k}\left(\begin{array}{c}
p-1 \\
\delta p+p-1-d-k
\end{array}\right)\left(\begin{array}{c}
2 j \\
k
\end{array}\right) \\
\equiv & \sum_{j=0}^{p-1}(-1)^{j} \sum_{\delta p-d \leqslant k<\delta p+p-d} 2^{k}\left(\begin{array}{c}
2 j \\
k
\end{array}\right)=\sum_{j=0}^{p-1}(-1)^{j} \sum_{t=0}^{p-1} 2^{\delta p-d+t}\left(\begin{array}{c}
2 j \\
\delta p-d+t
\end{array}\right) \\
\equiv & 2^{\delta-d} \sum_{s=0}^{p-1}(-1)^{s} \sum_{t=0}^{p-1} 2^{t}\left(\begin{array}{c}
2 s \\
\delta p-d+t
\end{array}\right)(\bmod p) .
\end{aligned}
$$

Therefore

$$
\begin{gathered}
\sum_{\delta p-d \leqslant 3 k \leqslant \delta p+p-1-d} 2^{k}\left(\begin{array}{c}
3 k+d \\
k
\end{array}\right) \\
\equiv(-2)^{\delta-d} \sum_{s=0}^{p-1}(-1)^{s} \sum_{t=0}^{p-1} 2^{t}\left(\begin{array}{c}
2 s \\
\delta p-d+t
\end{array}\right)(\bmod p) .
\end{gathered}
$$

Recall that $d \in\{0,1\}$. We have

$$
\begin{aligned}
& \sum_{s=0}^{p-1}(-1)^{s} \sum_{t=0}^{p-1} 2^{t}\left(\begin{array}{c}
2 s \\
\delta p-d+t
\end{array}\right) \\
= & \sum_{s=0}^{p-1}(-1)^{s} \sum_{t=-d}^{p-1-d} 2^{d+t}\left(\begin{array}{c}
2 s \\
\delta p+t
\end{array}\right) \\
= & \sum_{s=0}^{p-1}(-1)^{s}\left(\sum_{t=0}^{p-1} 2^{d+t}\left(\begin{array}{c}
2 s \\
\delta p+t
\end{array}\right)+d\left(\left(\begin{array}{c}
2 s \\
\delta p-1
\end{array}\right)-2^{p}\left(\begin{array}{c}
2 s \\
\delta p+p-1
\end{array}\right)\right)\right) \\
= & 2^{d} \sum_{s=0}^{p-1} \sum_{t=0}^{p-1}(-1)^{s} 2^{t}\left(\begin{array}{c}
2 s \\
\delta p+t
\end{array}\right)+d \sum_{s=0}^{p-1}(-1)^{s}\left(\left(\begin{array}{c}
2 s \\
\delta p-1
\end{array}\right)-2^{p}\left(\begin{array}{c}
2 s \\
\delta p+p-1
\end{array}\right)\right)
\end{aligned}
$$

and hence

$$
\begin{aligned}
& (-1)^{d+\delta} \sum_{\delta p-d \leqslant 3 k \leqslant \delta p+p-1-d} 2^{k}\left(\begin{array}{c}
3 k+d \\
k
\end{array}\right)-2^{\delta} \sum_{s=0}^{p-1} \sum_{t=0}^{p-1}(-1)^{s} 2^{t}\left(\begin{array}{c}
2 s \\
\delta p+t
\end{array}\right) \\
\equiv & d 2^{\delta-d} \sum_{s=0}^{p-1}(-1)^{s}\left(\left(\begin{array}{c}
2 s \\
\delta p-1
\end{array}\right)-2\left(\begin{array}{c}
2 s \\
\delta p+p-1
\end{array}\right)\right) \\
\equiv & d 2^{\delta-1}(3 \delta-2) \sum_{s=0}^{p-1}(-1)^{s}\left(\begin{array}{c}
2 s \\
p-1
\end{array}\right) \equiv d(3 \delta-2) 2^{\delta-1}(-1)^{(p-1) / 2}(\bmod p) .
\end{aligned}
$$

Since

$$
\sum_{s=0}^{p-1}(-1)^{s} \sum_{t=0}^{p-1} 2^{t}\left(\begin{array}{c}
2 s \\
\delta p+t
\end{array}\right) \equiv \frac{4-\delta}{10}+\frac{3}{10}(2-3 \delta)(-1)^{(p-1) / 2}(\bmod p)
$$


by Lemma 2.2 , we finally get

$$
\begin{aligned}
& \frac{(-1)^{d+\delta}}{2^{\delta}} \sum_{\delta p-d \leqslant 3 k \leqslant \delta p+p-1-d} 2^{k}\left(\begin{array}{c}
3 k+d \\
k
\end{array}\right) \\
\equiv & \frac{4-\delta}{10}+\frac{3}{10}(2-3 \delta)(-1)^{(p-1) / 2}+\frac{d}{2}(3 \delta-2)(-1)^{(p-1) / 2} \\
\equiv & \frac{4-\delta}{10}+\frac{(3 \delta-2)(5 d-3)}{10}(-1)^{(p-1) / 2}(\bmod p) .
\end{aligned}
$$

This proves (3.1).

Proof of Theorem 1.2. Let $d \in\{0,1\}$. If $(2 p-d) / 3 \leqslant k \leqslant p-1$, then $2 k+d+1 \leqslant$ $2 k+2 \leqslant 2 p \leqslant 3 k+d$ and hence

$$
\left(\begin{array}{c}
3 k+d \\
k
\end{array}\right)=\frac{(3 k+d) \cdots(2 k+d+1)}{k !} \equiv 0(\bmod p) .
$$

Therefore

$$
\sum_{2 p-d \leqslant 3 k \leqslant 3 p-3} 2^{k}\left(\begin{array}{c}
3 k+d \\
k
\end{array}\right) \equiv 0(\bmod p) .
$$

With the help of Theorem 3.1, we have

$$
\begin{aligned}
\sum_{k=0}^{p-1} 2^{k}\left(\begin{array}{c}
3 k+d \\
k
\end{array}\right) & \equiv \sum_{-d \leqslant 3 k \leqslant 2 p-1-d} 2^{k}\left(\begin{array}{c}
3 k+d \\
k
\end{array}\right) \\
& \equiv \sum_{\delta=0}^{1} \sum_{\delta p-d \leqslant 3 k \leqslant \delta p+p-1-d} 2^{k}\left(\begin{array}{c}
3 k+d \\
k
\end{array}\right) \\
& \equiv \sum_{\delta=0}^{1}(-1)^{d}(-2)^{\delta}\left(\frac{4-\delta}{10}+\frac{(3 \delta-2)(5 d-3)}{10}(-1)^{(p-1) / 2}\right) \\
& \equiv \frac{(-1)^{d-1}}{5}\left(1+(10 d-6)(-1)^{(p-1) / 2}\right)(\bmod p) .
\end{aligned}
$$

This yields (1.3) and (1.4). We are done.

\section{Proof of Theorem 1.3}

Proof of Theorem 1.3. Obviously (1.5) holds for $p=2,3$. Below we assume $p>3$.

Let $\delta \in\{0,1\}$. Applying (2.1) with $m=p+\delta p$ and $n=p$ we get

$$
\begin{aligned}
& 2^{p} \sum_{k=0}^{p}(-2)^{k}\left(\begin{array}{c}
p \\
p+\delta p-3 k
\end{array}\right)\left(\begin{array}{c}
3 k-\delta p \\
k
\end{array}\right) \\
= & (-1)^{\delta+1} \sum_{j=0}^{p}\left(\begin{array}{c}
p \\
j
\end{array}\right) \sum_{k=0}^{p+\delta p}(-2)^{k}\left(\begin{array}{c}
p \\
p+\delta p-k
\end{array}\right)\left(\begin{array}{c}
2 j \\
k
\end{array}\right) .
\end{aligned}
$$


Observe that

$$
\begin{aligned}
& \sum_{k=0}^{p}(-2)^{k}\left(\begin{array}{c}
p \\
p+\delta p-3 k
\end{array}\right)\left(\begin{array}{c}
3 k-\delta p \\
k
\end{array}\right) \\
= & \sum_{\delta p \leqslant 3 k \leqslant p+\delta p-1}(-2)^{k}\left(\begin{array}{c}
p \\
3 k-\delta p
\end{array}\right)\left(\begin{array}{c}
3 k-\delta p \\
k
\end{array}\right) \\
= & 1-\delta+\sum_{\delta p<3 k<p+\delta p}(-2)^{k}\left(\begin{array}{c}
p \\
3 k-\delta p
\end{array}\right)\left(\begin{array}{c}
3 k-\delta p \\
k
\end{array}\right) .
\end{aligned}
$$

For $j=1, \ldots, p-1$ clearly

$$
\left(\begin{array}{l}
p \\
j
\end{array}\right)=\frac{p}{j}\left(\begin{array}{l}
p-1 \\
j-1
\end{array}\right) \equiv p \frac{(-1)^{j-1}}{j}\left(\bmod p^{2}\right)
$$

Thus

$$
\begin{aligned}
& \sum_{\delta p<3 k<p+\delta p}(-2)^{k}\left(\begin{array}{c}
p \\
3 k-\delta p
\end{array}\right)\left(\begin{array}{c}
3 k-\delta p \\
k
\end{array}\right) \\
\equiv & \sum_{\delta p<3 k<p+\delta p}(-2)^{k} p \frac{(-1)^{3 k-\delta p-1}}{3 k-\delta p}\left(\begin{array}{c}
3 k-\delta p \\
k
\end{array}\right) \\
\equiv & (-1)^{\delta+1} \sum_{\delta p<3 k<p+\delta p}(-2)^{k} p \frac{(-1)^{k}}{3 k}\left(\begin{array}{c}
(3 k-\delta p)+\delta p \\
k
\end{array}\right) \\
\equiv & (-1)^{\delta+1} \frac{p}{3} \sum_{\delta p<3 k<p+\delta p} \frac{2^{k}}{k}\left(\begin{array}{c}
3 k \\
k
\end{array}\right)\left(\bmod p^{2}\right) .
\end{aligned}
$$

Notice that

$$
\begin{aligned}
& \sum_{j=0}^{p}\left(\begin{array}{l}
p \\
j
\end{array}\right) \sum_{k=0}^{p+\delta p}(-2)^{k}\left(\begin{array}{c}
p \\
p+\delta p-k
\end{array}\right)\left(\begin{array}{c}
2 j \\
k
\end{array}\right) \\
= & \sum_{\delta p \leqslant 2 j \leqslant 2 p}\left(\begin{array}{l}
p \\
j
\end{array}\right) \sum_{k=\delta p}^{p+\delta p}(-2)^{k}\left(\begin{array}{c}
p \\
k-\delta p
\end{array}\right)\left(\begin{array}{c}
2 j \\
k
\end{array}\right) \\
= & \sum_{\delta p<2 j<2 p}\left(\begin{array}{c}
p \\
j
\end{array}\right) \sum_{k=\delta p}^{p+\delta p}(-2)^{k}\left(\begin{array}{c}
p \\
k-\delta p
\end{array}\right)\left(\begin{array}{c}
2 j \\
k
\end{array}\right) \\
& +\sum_{2 j \in\{\delta p, 2 p\}}\left(\begin{array}{c}
p \\
j
\end{array}\right) \sum_{k=\delta p}^{p+\delta p}(-2)^{k}\left(\begin{array}{c}
p \\
k-\delta p
\end{array}\right)\left(\begin{array}{c}
2 j \\
k
\end{array}\right) .
\end{aligned}
$$


Clearly

$$
\begin{aligned}
& \sum_{\delta p<2 j<2 p}\left(\begin{array}{l}
p \\
j
\end{array}\right) \sum_{k=\delta p}^{p+\delta p}(-2)^{k}\left(\begin{array}{c}
p \\
k-\delta p
\end{array}\right)\left(\begin{array}{c}
2 j \\
k
\end{array}\right) \\
\equiv & \sum_{\delta p<2 j<2 p}\left(\begin{array}{c}
p \\
j
\end{array}\right)\left((-2)^{\delta p}\left(\begin{array}{c}
p \\
0
\end{array}\right)\left(\begin{array}{c}
2 j \\
\delta p
\end{array}\right)+(-2)^{p+\delta p}\left(\begin{array}{l}
p \\
p
\end{array}\right)\left(\begin{array}{c}
2 j \\
p+\delta p
\end{array}\right)\right) \\
\equiv & \sum_{\delta p<2 j<2 p}\left(\begin{array}{c}
p \\
j
\end{array}\right)(-2)^{\delta p}\left(\begin{array}{c}
2 j-\delta p \\
0
\end{array}\right) \\
& +(1-\delta) \sum_{p<2 j<2 p}\left(\begin{array}{c}
p \\
j
\end{array}\right)(-2)^{p+\delta p}\left(\begin{array}{c}
2 j-p \\
p-p
\end{array}\right)(\text { by Lucas' congruence }) \\
\equiv & (-2)^{\delta} 2^{1-\delta}\left(2^{p-1}-1\right)+(1-\delta)(-2)^{1+\delta}\left(2^{p-1}-1\right) \\
\equiv & (-1)^{\delta} \delta\left(2^{p}-2\right)=-\delta\left(2^{p}-2\right)\left(\bmod p^{2}\right) .
\end{aligned}
$$

(Note that $\delta \in\{0,1\}$ and $2 \sum_{p / 2<j<p}\left(\begin{array}{l}p \\ j\end{array}\right)=\sum_{j=1}^{p-1}\left(\begin{array}{l}p \\ j\end{array}\right)=2^{p}-2$.) Also,

$$
\sum_{2 j=\delta p}\left(\begin{array}{l}
p \\
j
\end{array}\right) \sum_{k=\delta p}^{p+\delta p}(-2)^{k}\left(\begin{array}{c}
p \\
k-\delta p
\end{array}\right)\left(\begin{array}{c}
2 j \\
k
\end{array}\right)=(1-\delta) \sum_{k=0}^{p}(-2)^{k}\left(\begin{array}{l}
p \\
k
\end{array}\right)\left(\begin{array}{l}
0 \\
k
\end{array}\right)=1-\delta
$$

and

$$
\begin{aligned}
& \sum_{2 j=2 p}\left(\begin{array}{c}
p \\
j
\end{array}\right) \sum_{k=\delta p}^{p+\delta p}(-2)^{k}\left(\begin{array}{c}
p \\
k-\delta p
\end{array}\right)\left(\begin{array}{c}
2 j \\
k
\end{array}\right) \\
\equiv & \sum_{k \in\{\delta p, p+\delta p\}}(-2)^{k}\left(\begin{array}{c}
p \\
k-\delta p
\end{array}\right)\left(\begin{array}{c}
2 p \\
k
\end{array}\right) \\
\equiv & (-2)^{\delta p}\left(\begin{array}{l}
2 \\
\delta
\end{array}\right)+(-2)^{p+\delta p}\left(\begin{array}{c}
2 \\
1+\delta
\end{array}\right)=4^{\delta p}-2^{p+1}\left(\bmod p^{2}\right) .
\end{aligned}
$$

(Recall that $\frac{1}{2}\left(\begin{array}{c}2 p \\ p\end{array}\right)=\left(\begin{array}{c}2 p-1 \\ p-1\end{array}\right) \equiv 1\left(\bmod p^{3}\right)$ by the Wolstenholme congruence (cf. $\mathrm{Gr}$ or [HT]).)

Combining the above with (4.1), we have

$$
\begin{aligned}
& 2^{p}\left(1-\delta+(-1)^{\delta+1} \frac{p}{3} \sum_{\delta p<3 k<p+\delta p} \frac{2^{k}}{k}\left(\begin{array}{c}
3 k \\
k
\end{array}\right)\right) \\
\equiv & (-1)^{\delta+1}\left(\delta\left(2-2^{p}\right)+1-\delta+4^{\delta p}-2^{p+1}\right)\left(\bmod p^{2}\right) .
\end{aligned}
$$

Setting $\delta=0$ and $\delta=1$ respectively, we obtain

$$
2^{p}-2^{p} \frac{p}{3} \sum_{0<3 k<p} \frac{2^{k}}{k}\left(\begin{array}{c}
3 k \\
k
\end{array}\right) \equiv 2^{p+1}-2\left(\bmod p^{2}\right)
$$

and

$$
2^{p} \frac{p}{3} \sum_{p<3 k<2 p} \frac{2^{k}}{k}\left(\begin{array}{c}
3 k \\
k
\end{array}\right) \equiv 2-2^{p}+4^{p}-2^{p+1}\left(\bmod p^{2}\right) .
$$

It follows that

$$
\frac{2}{3} p \sum_{0<3 k<2 p} \frac{2^{k}}{k}\left(\begin{array}{c}
3 k \\
k
\end{array}\right) \equiv 4^{p}-4 \cdot 2^{p}+4=\left(2^{p}-2\right)^{2} \equiv 0\left(\bmod p^{2}\right) .
$$


If $2 p \leqslant 3 k<3 p$, then

$$
\left(\begin{array}{c}
3 k \\
k
\end{array}\right)=\frac{3 k \cdots(2 k+1)}{k !} \equiv 0(\bmod p) .
$$

Therefore

$$
\sum_{k=1}^{p-1} \frac{2^{k}}{k}\left(\begin{array}{c}
3 k \\
k
\end{array}\right)=\sum_{0<3 k<2 p} \frac{2^{k}}{k}\left(\begin{array}{c}
3 k \\
k
\end{array}\right)+\sum_{2 p \leqslant 3 k<3 p} \frac{2^{k}}{k}\left(\begin{array}{c}
3 k \\
k
\end{array}\right) \equiv 0(\bmod p) .
$$

This completes the proof of Theorem 1.3.

\section{REFERENCES}

[Gr] A. Granville, Arithmetic properties of binomial coefficients. I. Binomial coefficients modulo prime powers, in: Organic Mathematics (Burnaby, BC, 1995), 253-276, CMS Conf. Proc., 20, Amer. Math. Soc., Providence, RI, 1997. MR.1483922 (99h:11016)

[HT] C. Helou and G. Terjanian, On Wolstenholme's theorem and its converse, J. Number Theory 128 (2008), 475-499. MR2389852 (2008k:11003)

[HS] H. Hu and Z.-W. Sun, An extension of Lucas' theorem, Proc. Amer. Math. Soc. 129 (2001), 3471-3478. MR1860478 (2002i:11019)

[PS] H. Pan and Z.-W. Sun, A combinatorial identity with application to Catalan numbers, Discrete Math. 306 (2006), 1921-1940. MR2251572 (2007d:05018)

[St] R. P. Stanley, Enumerative Combinatorics, Vol. 2, Cambridge Univ. Press, Cambridge, 1999. MR 1676282 (2000k:05026)

[ST1] Z.-W. Sun and R. Tauraso, On some new congruences for binomial coefficients, Acta Arith., to appear. http://arxiv.org/abs/0709.1665.

[ST2] Z.-W. Sun and R. Tauraso, New congruences for central binomial coefficients, preprint, http://arxiv.org/abs/0805.0563.

Department of Mathematics, Nanjing University, Nanjing 210093, People's Republic OF CHINA

E-mail address: zhaolilu@gmail.com

Department of Mathematics, Nanjing University, Nanjing 210093, People's Republic OF CHINA

E-mail address: haopan79@yahoo.com.cn

Department of Mathematics, Nanjing University, Nanjing 210093, People's Republic OF CHINA

E-mail address: zwsun@nju.edu.cn 